\title{
Programa de atención en dificultades perceptivas en estudiantes del nivel primario, Huancayo
}

\author{
Program of attention in perceptive difficulties of \\ primary students in Huancayo
}

\author{
Rocío Milagros Coz Apumayta ${ }^{1, *}$ \\ ${ }^{1}$ Escuela Académico Profesional de Psicología \\ Facultad de Humanidades, Universidad Continental
}

\section{RESUMEN}

El objetivo fue analizar la influencia que genera el programa atención en el desarrollo perceptivo en las estudiantes de la institución educativa $N^{\circ} 30054$ "Santa Maria Reyna" Huancayo. Para lo cual se planteo la hipótesis, el programa atención influye favorable y significativamente en el desarrollo perceptivo en estudiantes de la institución educativa No 30054 Santa María Reyna Huancayo. La investigación utilizó un diseño pre experimental, la muestra fue censal estando conformada por 184 estudiantes del 3ro. de primaria, cuyas edades oscilaban entre los 8 y 9 años, Se utilizó el test de Percepción de diferencias de L.L. Thurstone y M. Yela. Los resultados corresponden por lo tanto que el nivel de significación sig. $=0,000$, es menor que el $\alpha=0,05$, lo que permite rechazar la hipótesis nula de igualdad de medias de la prueba del pre-test y postest, llegando a la conclusión que el programa atención genera una influencia favorable con un nivel de significación unilateral del $5 \%$ en el desarrollo perceptivo en estudiantes de la Institución educativa N³0054 Santa María Reyna Huancayo. Lo cual nos lleva a afirmar, que el cerebro humano, está distribuido de tal manera que actúa con rapidez, para elegir una serie de estímulos rápidamente, y elegir un estímulo entre varias opciones, y si esta es potencializada mejorara tanto en su rapidez perceptiva así como su inteligencia espacial, obteniéndose mejores resultados en el proceso de aprendizaje.

Palabras claves: Atención, percepción, rapidez perceptiva, inteligencia espacial.

\begin{abstract}
The objective was to analyze the influence generated by the attention program on the perceptive development in the students of the educational institution N 30054 "Santa Maria Reyna" Huancayo. For which the hypothesis was raised, the attention program has a favorable and significant influence on the perceptive development in students of the educational institution N ${ }^{\circ} 30054$ Santa Maria Reyna Huancayo. The research used a pre experimental design, the sample was census being comprised of 184 students of the 3rd. of primary school, whose ages ranged between 8 and 9 years, the Perception of differences of L.L test was used. Thurstone and M. Yela. The results therefore correspond to the significance level sig $=0.000$, which is less than $\alpha$ $=0.05$, which allows rejecting the null hypothesis of equality of means of the pre-test and post-test, reaching the conclusion that the attention program generates a favorable influence with a level of unilateral significance of $5 \%$ in the perceptive development in students of the Educational Institution N 30054 Santa María Reyna Huancayo. Which leads us to affirm, that the human brain is distributed in such a way that it acts quickly, to choose a series of stimuli quickly, and choose a stimulus between several options, and if this is potentialized it will improve both its perceptive speed and as its spatial intelligence, obtaining better results in the learning process.
\end{abstract}

Keywords: Attention, perception, perception quickness, space intelligence.

Historial del artículo:

Recibido, 04 de marzo 2017; aceptado, 10 de noviembre de 2017; disponible en línea, 05 de enero de 2018

* Doctora en Educación, Magister en Psicología Educativa, Licenciada en Psicología. Docente de la Universidad Continental Huancayo, Perú. Correo: rcoz@continental.edu.pe 


\section{INTRODUCCIÓN}

Enmarcados dentro de la era del conocimiento, el avance tecnológico, donde los fundamentos educativos apuntan a resolver las necesidades planteadas, que exige una sociedad meramente competitiva e individualizada, donde poco a poco nos volvemos fríos y calculadores incapaces de sentir por la otra persona por el solo hecho de ganar y salir victorioso a nivel del rendimiento escolar, la presente investigación se presenta como una posibilidad de cambio, planteando un trabajo más en conjunto, y no limitado.

El informe presentado por el MINEDU de la evaluación ECE 2016, de la UGEL JUNIN, podemos observar los resultados obtenidos por el 4to. grado de primaria, en las evaluaciones realizadas en las áreas de Comunicación donde se evaluaron las capacidades de: recuperación de información, infiere el significado del texto, reflexiona sobre la forma, el contenido y el contexto se observa en los resultados a nivel de sexo un $35.3 \%$ rendimiento satisfactorio en mujeres, en comparación con los hombres que logran un nivel de satisfacción del $36.0 \%$, y a nivel de Gestión se observa un $35.7 \%$ de satisfacción en colegios estatales, en comparación a gestión privada que logra un nivel de satisfacción $48.2 \%$.

A nivel de Matemática, se evaluó las capacidades de: matematiza situaciones, comunica y representa ideas matemáticas, elabora y usa estrategias, razona y argumenta generando ideas matemáticas, se observó los siguientes resultados a nivel de sexo se observa un $35.9 \%$ de satisfacción en varones comparación con las mujeres quienes logran un nivel de satisfacción de un $29,6 \%$ de satisfacción, y a nivel de gestión la Escuela Estatal Urbana llega a una satisfacción de $35.1 \%$ y el No Estatal logra un 35.8\% de satisfacción, esto es una clara visión de que ser mujer y de colegio estatal, se demuestra ciertas desventajas a nivel de su rendimiento.

Así mismo la UNESCO (2011), tiene como uno de sus objetivos pedagógicos el de "construir sistemas educativos eficaces en el mundo entero, que abarquen desde la primera infancia hasta la edad adulta".

Por otro lado en una encuesta realizada a las estudiantes de la Institución educativa №30054 Santa María Reyna Huancayo se observa que un $50.27 \%$ (92 estudiantes) respondieron que en clase muestran una escasa atención, ubicándose en la categoría de Mucho, refiriendo que están observando a las maestras, pero sus pensamientos se encuentran muy lejos, esto coincide con la encuesta realizada a los maestros del tercero de primaria quienes en un $80 \%$ ubican a sus estudiantes en la categoría bastante con respecto a que muestran escasa atención, lo cual nos indicaría que los maestros perciben que las estudiantes cuando dictan sus clases, no se encuentran atentas a las instrucciones por parte de ellos.

Con respecto al items si tienen dificultades en su orientación espacial, se puede observar que un $31.14 \%$ de las estudiantes respondieron que presentan poca dificultades en su orientación espacial. Por el contrario sus maestros en un $60 \%$ respondieron que las estudiantes muestran mucha dificultades a nivel de su orientación espacial, como podemos analizar los maestros, en el proceso de enseñanza aprendizaje van visualizando los avances individuales que van presentando sus estudiantes, y al realizar un análisis más exhaustivo, van comparando rendimientos individuales de sus estudiantes y de modo general de su aula, si logran los parámetros programados en su proceso de enseñanza-aprendizaje.

De acuerdo al Proyecto Educativo Nacional (PEN), mencionado por la I.E. Santa María Reyna (2016); todas las estudiantes deben lograr competencias fundamentales para su desarrollo personal y el progreso e integración nacional. Toda institución es acogedora e integradora, donde se enseña bien y se hace con éxito. Así mismo contamos con maestros bien preparados que ejercen profesionalmente la docencia. Pero se requiere de profesionales en psicología a nivel de la primaria financiado por el Ministerio de Educación que favorezca y complemente el trabajo del docente en el aula.

El Proyecto Educativo Regional (PER) mencionado por la I.E. Santa María Reyna (2016), la cual garantiza el derecho a una educación pública gratuita que atienda a las necesidades e intereses de las estudiantes, así como sus demandas y expectativas de la sociedad regional, nacional y global. Lo cual indicaría que la atención integral es ver a la estudiante en todos sus aspectos que la lleven a un buen desempeño escolar y que le permita un crecimiento personal donde se dé cuenta que avanza bien en sus aprendizajes y que le permite desarrollarse adecuadamente.

\section{Problema general}

¿Cómo influye la aplicación del programa atención en el desarrollo perceptivo en las estudiantes de la institución educativa №30054 Santa María Reyna Huancayo?

Los problemas específicos son:

- ¿De qué manera el programa de atención influye en la dimensión de la rapidez perceptiva en las estudiantes de la institución educativa $N^{\circ} 30054$ Santa María Reyna Huancayo?

- ¿De qué manera el programa atención influye en la dimensión de inteligencia espacial en las 
estudiantes de la institución educativa №30054 Santa María Reyna Huancayo?

\section{Objetivo general}

Analizar la influencia que genera el programa atención en el desarrollo perceptivo en las estudiantes de la institución educativa N $N^{\circ} 30054$ Santa María Reyna Huancayo.

Los objetivos específicos son:

- Determinar la influencia del programa atención en el desarrollo de la dimensión de rapidez perceptiva en estudiantes de la institución educativa N $N^{\circ} 30054$ Santa María Reyna- Huancayo.

- Analizar la influencia del programa atención en el desarrollo de la dimensión de inteligencia espacial en las estudiantes de la institución educativa Santa María Reyna Huancayo.

\section{Hipótesis general}

El programa atención influye favorablemente y significativamente en el desarrollo perceptivo en estudiantes de la institución educativa №30054 Santa María Reyna Huancayo.

Las hipótesis específicas son:

- El programa de atención influye favorable y significativamente en el desarrollo de la dimensión de rapidez perceptiva en estudiantes de la institución educativa N 30054 Santa María Reyna Huancayo.

- El programa de atención influye favorable y significativamente en el desarrollo de la dimensión de inteligencia espacial en estudiantes de la institución educativa № 30054 Santa María Reyna Huancayo.

\section{Justificación teórica}

Wiilliam James, mencionado por (Coon, 1998), refiere que para el nuevo ser aun pequeño el mundo totalmente un desconcierto reluciente y de murmullo, en la cual a medida que empiece asociarlo con su significado encontrara la interpretación de lo que sucede. Lo cual nos indica que cada ser cuando va ingresando año tras año en sus aprendizajes, le va ir dando un significado y las experiencias vividas le permitirán entenderse así misma sobre sus progresos, o dificultades, cabe mencionar que aun los pequeños cada vez que van ascendiendo los grados le permite realizar un análisis a profundidad en sus desempeños, si son esperados o con dificultad.

Según Frosting (1994), refiere que la percepción es la capacidad de identificar y discernir estímulos visuales y de analizarlos, ya sea esto al vincularlos con acontecimientos anteriores.

La percepción es sin duda la interpretación de lo observado, que va a responder a una gama de estímulos externos, dentro de una configuración de la totalidad. La facultad que tiene la vista de reconocer e interpretar los diferentes estímulos visuales y analizarlos, a partir de experiencias anteriores permite el reconocimiento e identificación de lo aprendido.

\section{Justificación práctica}

Por medio de la investigación podremos observar como el programa atención mejora los niveles perceptivos en las estudiantes de la institución educativa Santa N 30054 María Reyna Huancayo - 2016, de este modo contribuya con el crecimiento personal y desarrollo de habilidades en las estudiantes y prever de este modo un trabajo preventivo que conlleven a un rendimiento académico esperado por los maestros y de esta manera plantear programas estratégicos de intervención temprana con estudiantes, que a su vez permitirán tener una autoimagen positiva de ellas mismas, que perduren a través del tiempo.

\section{Antecedentes}

Enmarcamos en los principios psicológicos, es sin duda que tenemos que referirnos, al momento en la cual la psicología se torna de un aspecto filosófico, a un periodo científico, referido por Coon (1998), dándose en 1879, donde se crea el primer laboratorio experimental en la Universidad de Leipzig, Alemania por Wilhelm Wundt, quien fue representante del estructuralismo, con su método la introspección analítica, cuyo objeto de estudio es la mente humana, las experiencias sensoriales, en las cuales las experimentaciones juegan un papel importante para la comprobación del conocimiento, a través de la experimentación y control de los estímulos adyacentes, así mismo tenemos que destacar a Más Wertheimer representante de la Gestalt, con su método introspección, métodos objetivos, cuyo objeto de estudio es la conciencia, la percepción, en la cual se funda la presente investigación; Así mismo debemos mencionar a Jean Piaget representante del cognitivismo, su método fue los métodos experimentales, y el objeto de estudio los procesos psíquicos; quien nos habla acerca de las etapas de la inteligencia.

Verdugo (2014), realiza la investigación Inteligencia espacial y su relación con el rendimiento académico en niños escolarizados de 10 a 12 años. Esta investigación se ubica dentro del paradigma cuantitativo, con un método y diseño transversal correlacional, no experimental, la cual se desarrolla con la participación 59 alumnos (29 niños y 30 niñas), estudiantes de quinto y sexto grado de primaria con edad de 10 a 12 
años respectivamente. En cuanto a la relación existente entre el desarrollo de las habilidades espaciales y el rendimiento académico se afirma que existen relaciones significativas, ya que les permite reconocer las relaciones existentes entre los objetos de la realidad, las especies y la posibilidad de movimiento y la segunda es consecuencia de una mayor estructuración del espacio en la mente, lo que proporciona imágenes mentales más claras y detalladas de la realidad, lo que da la posibilidad de representar una semejanza grafica de información espacial, pues, a mejores imágenes mentales de los espacios a representar, mejores representaciones graficas de las mismas, lo cual se traduce a un mayor rendimiento cognitivo.
Castellanos (2015), intervención en el aula para la mejora de la atención y el rendimiento en el alumnado de segundo nivel de educación primaria: eficacia de la autoinstrucción y de la autobservación. La encuesta ha sido contestada por un total de 276 maestros/as en activo que, en el momento de responder la encuesta, se encontraron impartiendo la docencia en el primer ciclo de educación primaria, así como 71 alumnos.

El $91 \%$ de los maestros encuestados aseguran que han detectado en los últimos años un incremento de los problemas atencionales en los alumnos cuando realizan las tareas escolares. Este dato apunta a la línea de que en la actualidad se está produciendo

Tabla 1

Programa de atención Rocio.

\begin{tabular}{|c|c|c|c|}
\hline Sesión & Denominación & Actividades & Tiempo \\
\hline $\mathrm{N}^{\circ} 1$ & "Iniciando" & $\begin{array}{l}\text { En las diferentes sesiones; se trabajo dinámicas } \\
\text { diversas de atención a través de canciones y }\end{array}$ & \\
\hline$N^{\circ} 2$ & $\begin{array}{l}\text { "Conociendo mis } \\
\text { habilidades" }\end{array}$ & $\begin{array}{l}\text { juegos. } \\
\text { Durante el desarrollo: Las actividades } \\
\text { denominadas: LAS }\end{array}$ & \\
\hline$N^{\circ} 3$ & $\begin{array}{l}\text { "Trabajando con } \\
\text { mi mente" }\end{array}$ & $\begin{array}{l}\text { CALENTAMIENTO, consistente en realizar } \\
\text { trabajos en hojas cuadriculadas, por espacio } \\
\text { de } 1,2 \text { o } 3 \text { minutos como maximo dandoles las }\end{array}$ & \\
\hline $\mathrm{N}^{\circ} 4$ & $\begin{array}{l}\text { "Observando } \\
\text { mejor" }\end{array}$ & $\begin{array}{l}\text { indicaciones de los reactivos a las estudiantes.La } \\
\text { siguiente actividad denominada: ATENTO CON } \\
\text { NUESTROS OIDOS, mencionando a las alumnas }\end{array}$ & \\
\hline $\mathrm{N}^{\circ} 5$ & $\begin{array}{l}\text { "Identificando } \\
\text { objeto" }\end{array}$ & $\begin{array}{l}\text { una lista de diez reactivos y luego procedian } \\
\text { a escribir lo que se recordaban trabajando } \\
\text { cuatro actividades, para cada sesión, estas }\end{array}$ & El tiempo \\
\hline No6 & $\begin{array}{l}\text { "Trabajando en } \\
\text { orden" }\end{array}$ & $\begin{array}{l}\text { actividades fueron variando en cada sesión; La } \\
\text { tercera actividad se denomino : ATENTO CON } \\
\text { NUESTRAS MENTES, esta actividad comprendia }\end{array}$ & $\begin{array}{l}\text { cada una de } \\
\text { las sesiones } \\
\text { son de } 90\end{array}$ \\
\hline$N^{\circ} 7$ & $\begin{array}{l}\text { "Observando } \\
\text { bien" }\end{array}$ & $\begin{array}{l}\text { dos tipos de trabajos, en las primeras las } \\
\text { estudiantes tenian que completar nombres } \\
\text { solicitados con una Inicial de una letra del }\end{array}$ & \\
\hline$N^{\circ} 8$ & $\begin{array}{l}\text { "Trabajando con } \\
\text { rapidez" }\end{array}$ & $\begin{array}{l}\text { abecedario, o de actividades de la vida cotidiana } \\
\text { y la otra actividad en la que trabajaban con un } \\
\text { material diseñado por la investigadora siendo : }\end{array}$ & \\
\hline $\mathrm{N}^{\circ} 9$ & $\begin{array}{l}\text { "Trabajando con } \\
\text { atención" }\end{array}$ & $\begin{array}{l}\text { Formas y colores, Uniendo partes en mi mente, } \\
\text { ubicando figuras igual al modelo encerrando en } \\
\text { un circulo figuras iguales, pintando las figuras }\end{array}$ & \\
\hline$N^{\circ} 10$ & $\begin{array}{l}\text { "Observando mi } \\
\text { avance" }\end{array}$ & $\begin{array}{l}\text { solicitadas, marcando con un aspa las figuras } \\
\text { indicadas, esta parte del trabajo tenia como } \\
\text { tiempo 1, } 2 \circ 3 \text { minutos de trabajo práctico. }\end{array}$ & \\
\hline
\end{tabular}

Fuente: de la investigadora

El perfil cognitivo de la inteligencia espacial en los niños y niñas que se destacan en este tipo de inteligencia está estrechamente ligadas a procesos de alto desarrollo como la percepción, meta cognición, memorización, abstracciones y expresión grafico-plástica. Reconocer y valorar y atender las habilidades espaciales que configuran la inteligencia espacial y brindar un marco de autorrealización y satisfacción escolar que influye fuertemente en la educación centrada en el individuo, lograra que el alumno desarrolle sus capacidades, conocimientos, necesidades e intereses que se adecuen con su perfil contribuyendo al desarrollo de sus inteligencias. problemas atencionales en las aulas y apoyan la realidad de trabajar la capacidad atencional de los alumnos, para mejorarla.

Lara (2004) investiga la utilización del ordenador para el desarrollo de la visualización espacial. La investigación es tipo experimental, Llegando a las siguientes conclusiones: hemos comprobado las mejoras producidas tanto en el razonamiento abstracto como en la visión espacial en los distintos grupos experimentales, los resultados son significativos. No nos atrevemos a firmar que los programas de ordenador sean los únicos responsables de las mejoras, ni siquiera podemos decir que tanto el 
razonamiento abstracto como la visión espacial sean habilidades exclusivas de la materia de educación plástica y visual. Cuando hablamos del desarrollo de capacidades lo tratamos como el resultado de un conjunto de intervenciones desde distintas áreas en el proceso cognitivo del alumno. Pero si podemos afirmar con cierto fundamento que intervenciones como la que nosotros hemos practicado ayudan al alumno en su proceso de aprendizaje.

Guevara (2012), realiza la investigación: Instrumento para el estudio de la percepción, su aplicación a la arquitectura y la lectura del espacio. Llegando a las siguientes conclusiones: el propósito principal ha sido confirmar y corroborar en todo sentido la hipótesis de que la percepción es "el enlace atemporal entre el hombre y el espacio, es el medio vinculante que hace posible la experiencia cognoscitiva y entendimiento de su mundo físico existente, que por medio del control de esta se adquiere la posibilidad de poder manipular si en las alumnas se potencializa las habilidades de rapidez perceptiva e inteligencia espacial, a traves de diferentes actividades de atención, se lograra un mejor desempeño en su rendimiento escolar y a la vez mejorara su autoconcepto y por consiguiente su autoestima, pues debemos recordar que la percepción es la puerta de ingreso para el proceso de enseñanza aprendizaje.

\section{MATERIAL Y MÉTODOS}

Como método general se tiene al método cientifico, como metodo especifico el experimental y estadistico. El diseño del estudio fue pre experimental (Sierra, 2007), y refiere que según Campbell y Stanley, este diseño es el más utilizado en los diseños experimentales básicos, porque accede a verificación de las variables externas indicadas ligadas al tiempo, a excepción

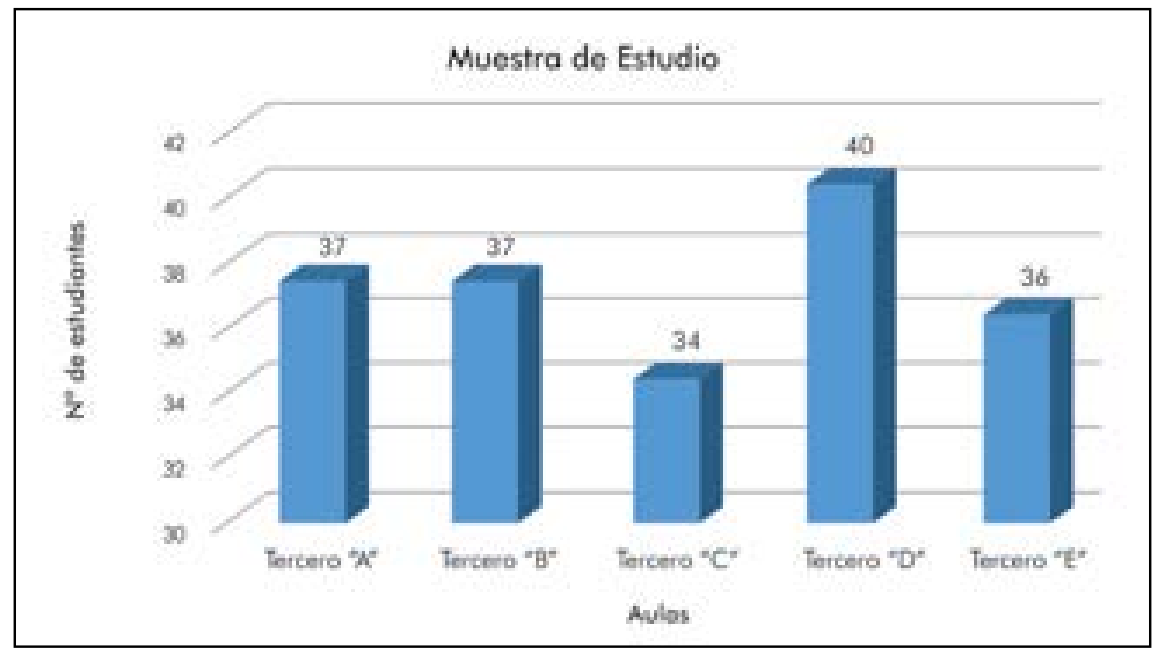

Figura 1. Muestra de estudio.

los sentidos, para un mejor control de los elementos y las variables que intervienen en el diseño y la creación del espacio. Se abordó en la primera sección de esta investigación el enfoque psicológico acerca de la percepción como parte de la demostración de la hipótesis de esta tesis, ya que esta disciplina la ha considerado dentro de sus principales motivos de estudio definiéndola en términos generales como el proceso cognitivo de la conciencia del hombre capaz de reconocer e interpretar sensaciones del mundo físico, en las que intervienen otros procesos psicológicos entre los que se encuentran el aprendizaje y la simbolización.

\section{Contribución}

La contribución realizada es la propuesta del "Programa de Atención Rocio", para mejorar las dificultades perceptivas en aula; En la tabla 1 podemos visualizar las diez sesiones realizadas. Considero que de la interacción entre la administración del test y la variable experimental, por lo que su validez interna es aceptable.

$\mathrm{G}: \mathrm{O}_{1} \times \mathrm{O}_{2}$

Donde:

$\mathrm{O}_{1}=$ Pre-test

$\mathrm{X}$ = Tratamiento, programa de atención

$\mathrm{O}_{2}=$ Post- Test

La población investigada son 1146 estudiantes de la Institución Educativa N 30054 Santa María Reyna Huancayo, 2016 población objeto de estudio.

La muestra lo constituyen 184 estudiantes que cursan el $3^{\circ}$ primaria, como se muestra en la figura 1 , muestra censal. 
Cómo técnicas de recolección de datos se utilizó el Test de percepción de diferencias de L. L. Thurstone y Yela, el objetivo es de explorar o medir las aptitudes para percibir rápida y correctamente, semejanzas y diferencias y patrones estimulantes parcialmente ordenadas, (60 ítems). La Validez: criterios factoriales, esta prueba ha sido estudiada en numerosos análisis factoriales. Los resultados radican que su varianza se distribuye principalmente entre los factores $\mathrm{P}$ (rapidez perceptiva) y S (inteligencia espacial, en los estudios de
Los datos que obtenidos del pre-test y el post-test fueron procesados mediante la estadística descriptiva, donde se utilizó un contraste de hipótesis de diferencias de medias, para datos apareados (Triola, 2012), de acuerdo al diseño de investigación se elaboraron las tablas y gráficos con sus respectivos análisis e interpretación, propias de una investigación, utilizando el programa estadístico SPSS versión 24.

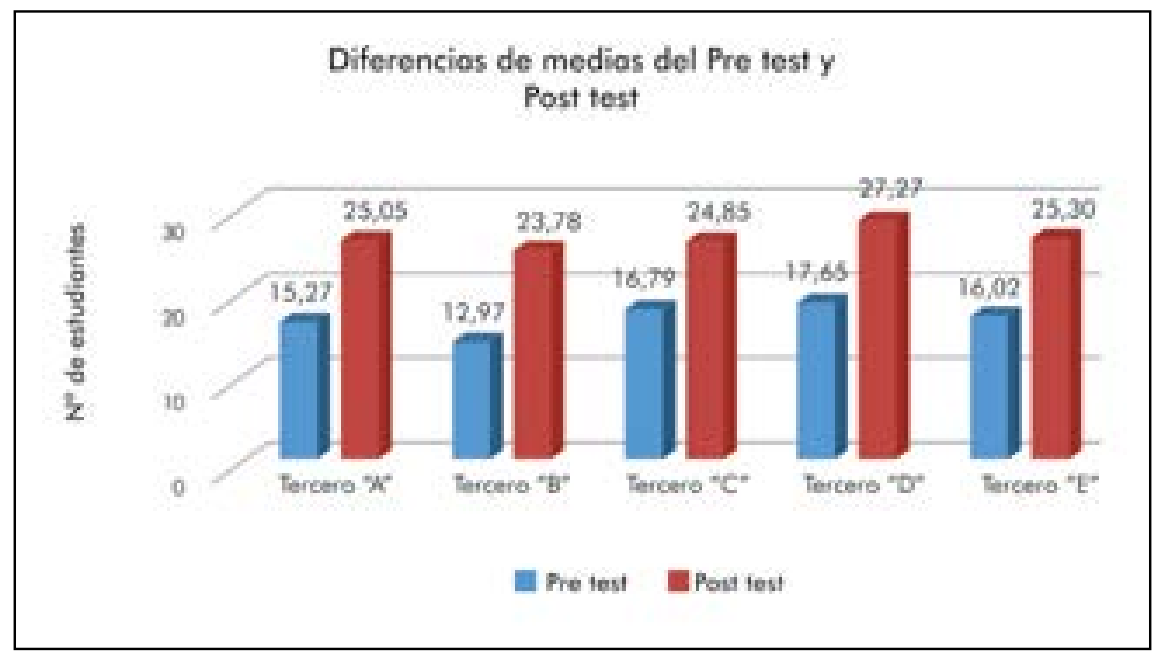

Figura 2, Diferencias de medias del Pre-test y Post test.

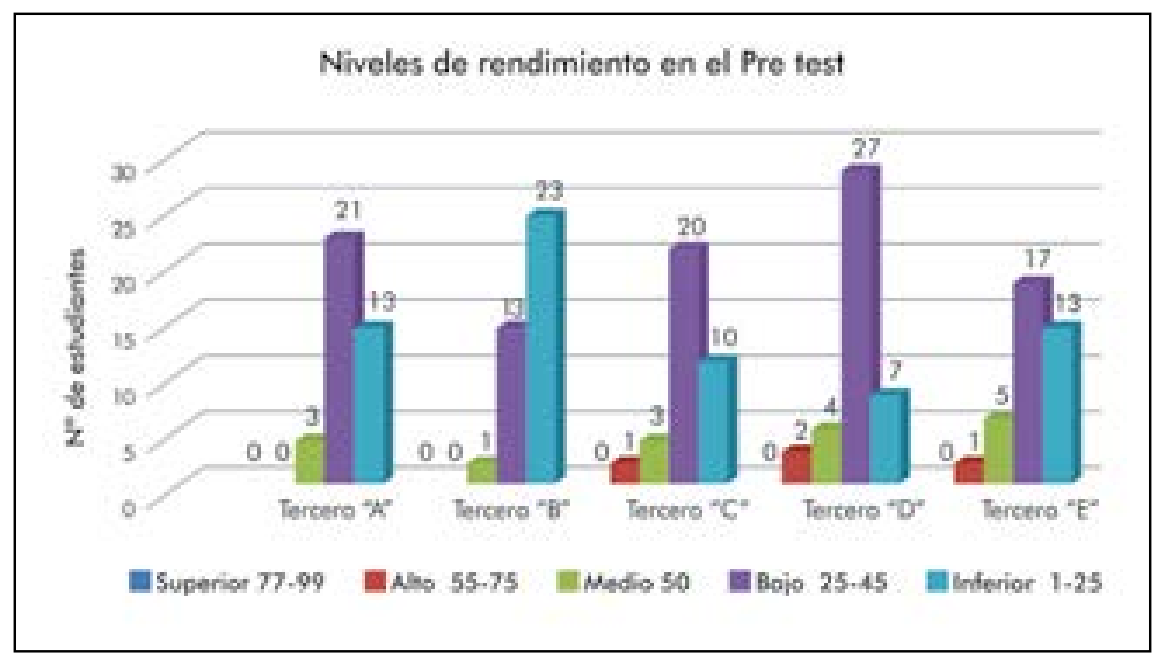

Figura 3, Niveles de Rendimiento en el Pre-test.

Thurstone aparece con una saturación en torno a 0,40 en factores de rapidez, y con saturación algo menores en factores espaciales. Por otro lado la confiabilidad del instrumento se obtuvo a través de la correlación ítem-test, comprobándose que todos los ítems mostraban correlaciones mayores de 20. Así mismo se utilizó el método de consistencia interna, la cual arrojo un estimado de 0.90 .
Los materiales utilizados en el "Programa de atención Rocío", fueron:

- Hojas impresas (2 hojas)

- 1 hoja cuadriculada

- Lápices

- Colores

- Reglas 


\section{RESULTADOS}

El primer paso fue obtener las diferencias de medias del Pre test y Post test por secciones, para lo cual lo presentamos en la figura 2.

Niveles de rendimiento del Pre test, como podemos observar en la figura 3, el rendimientos en las alumnas con el pre-test, se halla un alto \% entre los niveles de rendimiento bajo e inferior, y observándose una menor frecuencia de \% en los niveles medio y alto y no presentándose ningún nivel superior. atención en el desarrollo perceptivo en las estudiantes de la Institución Educativa № 30054 Santa María Reyna Huancayo, para ello se utilizó un contraste de hipótesis de diferencia de medias para datos apareados de acuerdo al diseño de la investigación, además porque las variables han sido cuantificadas (tabla 2).

Los resultados corresponden por lo tanto que el nivel de significación sig. $=0,000$, es menor que el $\alpha=0,05$, lo que permite rechazar la hipótesis nula de igualdad de medias de la prueba del pre-test y postest, llegando a la conclusión que el programa atención genera

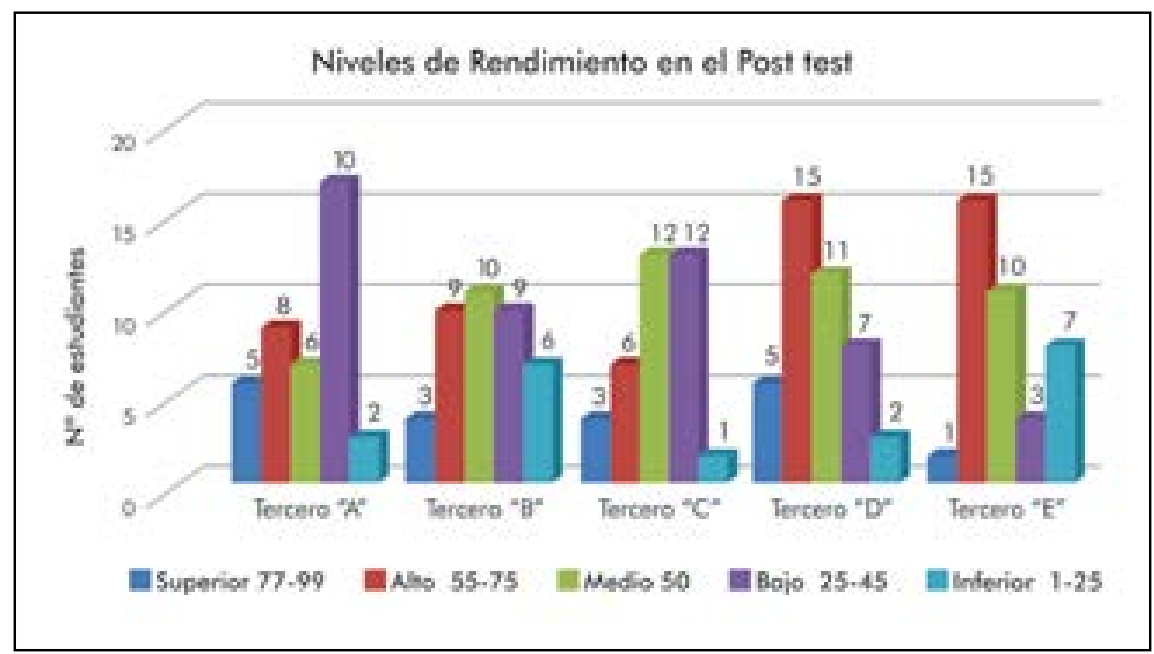

Figura 4. Niveles de Rendimiento en el Post test.

Tabla 2

Decisión estadística respecto a la hipótesis general influencia del programa atención en el desarrollo perceptivo.

\begin{tabular}{|c|c|c|c|c|c|c|c|c|c|}
\hline \multicolumn{10}{|c|}{ Prueba de muestras emparejadas } \\
\hline & & \multicolumn{5}{|c|}{ Diferencias emparejadas } & \multirow{3}{*}{$t$} & \multirow{3}{*}{ gl } & \multirow{3}{*}{$\begin{array}{c}\text { Sig. } \\
\text { (bilateral) }\end{array}$} \\
\hline & & \multirow[t]{2}{*}{ Media } & \multirow[t]{2}{*}{$\begin{array}{l}\text { Desviación } \\
\text { estándar }\end{array}$} & \multirow[t]{2}{*}{$\begin{array}{l}\text { Media } \\
\text { de error } \\
\text { estándar }\end{array}$} & \multicolumn{2}{|c|}{$\begin{array}{l}95 \% \text { de intervalo } \\
\text { de confianza de } \\
\text { la diferencia }\end{array}$} & & & \\
\hline & & & & & Inferior & Superior & & & \\
\hline $\begin{array}{c}\text { Par } \\
1\end{array}$ & $\begin{array}{l}\text { pretest - } \\
\text { postest }\end{array}$ & $-9,543$ & 6,485 & 0,478 & $-10,487$ & $-8,600$ & $-19,963$ & 183 & 0,000 \\
\hline
\end{tabular}

Fuente: Propia de la investigadora

Niveles de rendimiento del post test, en la figura 4, se puede observar una variabilidad en los resultados de niveles, evidenciándose la presencia del nivel Superior, el nivel Alto aumenta en su frecuencia, así como el nivel Medio; Se evidencia un decrecimiento en el nivel Bajo e Inferior.

\section{Proceso de prueba de hipótesis general}

La hipótesis general de la investigación trata de analizar la influencia que genera el programa de una influencia favorable con un nivel de significación unilateral del $5 \%$ en el desarrollo perceptivo en estudiantes de la Institución educativa N 30054 Santa María Reyna Huancayo.

\section{DISCUSIÓN}

La hipótesis general de la investigación planteada fue la de analizar la influencia que genera el programa de atención en el desarrollo perceptivo en las estudiantes de la Institución Educativa № 30054 Santa María Reyna 
Huancayo, se logró obtener un nivel de significancia unilateral del $5 \%$ si existe influencia del programa atención en el desarrollo perceptivo en estudiantes de la Institución Educativa N 30054 Santa María Reyna Huancayo. Según Goldstein (2005) considera a la percepción como un acontecimiento sensible y juicioso, lo cual nos indicaría que es susceptible de mejorar e ir en aumento progresivo, si es estimulado.

Haciendo referencia a Castellano (2015), Intervención en el aula, para mejorar la atención y el rendimiento en el alumnado de segundo nivel de educación primaria: Eficacia de la autoinstrucción y de la autoinformación, en sus conclusiones observa que un $91 \%$ de los maestros encuestados observaron que se han detectado en los últimos años un incremento de los problemas atencionales en los alumnos cuando realizan la tarea escolares, los cuatro grupos no presentan diferencias significativas en la variable atención medida a través de CARAS-R, aun así señala que tras la intervención las puntuaciones de los tres grupos experimentales en aula variables atencionales fueron superiores a las puntuaciones obtenidas por el grupo control, lo cual contrasta con nuestra investigación donde se observa el incremento de los niveles perceptivos en un mejora que se da con un nivel de significación unilateral del $5 \%$.

Más adelante refiere Castellano (2015) “... que los estudiantes a través de la autoevaluación de los estudiantes apuntan a una percepción significativa más positiva del propio nivel atencional por parte de los estudiantes de los grupos experimentales respecto al grupo control. Lo cual pude contrastar con la observación que fueron dando las estudiantes, cuando se le preguntaba cómo le iba ahora si habían observado un cambio en ellas, las cuales mencionaron que se habían vuelto más rápidas en el copiado y en realizar trabajos escolares.

La hipótesis especifica fue el programa de atención influye favorablemente y significativamente en la dimensión de rapidez perceptiva en las estudiantes de la Institución Educativa $N^{\circ} 30054$ Santa María Reyna Huancayo, logrando concluir que se rechaza la hipótesis nula y se acepta la hipótesis de investigación hallando un nivel de significación unilateral del 5\%, que el programa de atención si influye favorablemente y significativamente en el desarrollo de la dimensión de rapidez perceptiva en las estudiantes de la Institución educativa N 30054 Santa María Reyna Huancayo.

Según Coon (1998), refiere que las percepciones significativas están compuestas por figura y fondo, donde se debe entender que el cerebro está distribuido de tal manera que es capaz de elegir entre una serie de estímulos que llegan, un cierto número circunscritos que son convertidos en el eje del interés, las dificultades que puedan presentarse es que las personas pueden centrar su atención en elementos accesorios y secundarios que no son importantes, lo cual nos lleva a afirmar nuestra hipótesis, el cerebro humano, está distribuido de tal manera que actúa con rapidez, para elegir una serie de estímulos rápidamente, y elegir un estímulo entre varias opciones, así mismo Goldstein (2005) nos refiere que las conductas perceptuales, son las observaciones entendidas como una amplitud, para situar los diferentes elementos en jerarquías que les confiere una interpretación adecuada, si bien es cierto que se da una jerarquía a los elementos para la interpretación, esta no tiende a tardar en el ser humano es una actividad que se realiza rápidamente, en segundo y/o minutos.

Así mismo Vizcarra (2012) realiza la investigación organización perceptiva y escritura cursiva en el primer grado de primaria de tres instituciones educativas del Callao, donde arriba a las siguientes conclusiones, Existe relación significativa en la organización perceptual y la velocidad normal de ejecución en la calidad caligráfica de la escritura cursiva en los estudiantes del primer grado de educación primaria, lo que significa que los alumnos que tienen buena organización perceptiva tienen una buena calidad caligráfica en la escritura cursiva.

Existe relación significativa entre la organización perceptiva y la velocidad normal de ejecución en la calidad caligráfica de la escritura cursiva en los estudiantes del primer grado de educación primaria, lo que significa, que los alumnos que tienen una buena organización perceptiva también tienen una buena velocidad normal de ejecución al escribir en forma cursiva, Lo cual nos lleva a plantear que de acuerdo a nuestra investigación, que las estudiantes que desarrollan una mayor velocidad perceptiva, obtendrá mejores resultados en su proceso de aprendizaje, pues realizara una mejor discriminación de los estímulos.

La hipótesis especifica fue el programa de atención influye favorablemente y significativamente en el desarrollo de la dimensión de inteligencia espacial, logrando concluir que se rechaza la hipótesis nula, y se acepta la hipótesis de investigación en la que existe un nivel de significación unilateral del $5 \%$ que el programa de atención si influye favorable y significativamente en el desarrollo de la dimensión de inteligencia espacial en las estudiantes de la Institución educativa No 30054 Santa María Reyna Huancayo. Debemos indicar que desde Gardner, mencionado por Verdugo (2014), planteara la existencias de varias inteligencias, o puertas de ingreso del conocimiento, se produjo una gran revolución con respecto a las diferentes teorías plantean anteriormente, donde se daba solo la importancia de dos tipos de inteligencia, Gardner habla de ocho tipos de inteligencia las cuales son desarrolladas en cada individuo de diferente manera, en algunos más que otros, destacándose en 
las actividades en la cual un sujeto resalta más sus habilidades que le permite sobresalir entre varios.

Así mismo Verdugo (2014).realiza la investigación Inteligencia espacial y su relación con el rendimiento académico en niños escolarizados de 10 a 12 años. En el análisis de los resultados referidos al factor de inteligencia espacial, se rechaza la hipótesis nula y se acepta la hipótesis de investigación, en la cual se indica "el desarrollo de la inteligencia espacial determina el rendimiento académico en niños escolarizados de 10 a 12 años".

Más adelante refiere que el perfil cognitivo de la inteligencia espacial en los niños y niñas que se destaca en este tipo de inteligencia está estrechamente ligada a proceso de alto desarrollo como la percepción, meta cognición, memorización, abstracciones y expresiones grafico-plástica. Reconocer y valorar y atender las habilidades especiales que configuran la inteligencia espacial y brindar un marco de autorrealización y satisfacción escolar que influye fuertemente en la educación centrada en el individuo, lograra que el alumno desarrollo sus capacidades, conocimientos, necesidades e intereses que se adecuen con su perfil contribuyendo al desarrollo de sus inteligencias.

Cabe más que decir que estamos de acuerdo con la afirmaciones encontradas por Verdugo (2014) que con un programa que atienda y potencialice el desarrollo de la percepción y la memorización favorecerá eficazmente al alumno, si bien es cierto a través de la investigación se realizó trabajos grupales, donde se observó la potencializarían de las alumnas, así mismo se observó que un número más reducido tanto en su pre-test y luego en su post test el desarrollo obtenido era mínimo y que requieren de atención en su desarrollo perceptivo, pero más individualizado, pues las dificultades que suele estar presentando no son bien atendidos cuando estos se le dan forma grupal, pues su incremento es mínimo en lo que respecta a la inteligencia espacial trabajada.

Así mismo tenemos a Lara (2004), quien realiza la investigación; Utilización del ordenador para el desarrollo de la visualización espacial, donde utiliza programas didácticos diseñados para el ordenador llegando a las conclusiones siguientes ha comprobado las mejoras producidas tanto en el razonamiento abstracto como en la visión espacial en los distintos grupos experimentales, los resultados son significativos, donde refiere "No nos atrevemos a firmar que los programas de ordenador sean los únicos responsables de las mejoras, ni siquiera podemos decir que tanto el razonamiento abstracto como la visión espacial sean habilidades exclusivas de la materia de educación plástica y visual".
Por otro lado tenemos Huertas \& Otros (2010) donde realiza la investigación Las inteligencias múltiples y el aprendizaje en las diferentes áreas curriculares en los estudiantes del 4to. Y 5to. Ciclo de primaria del Colegio experimental "Víctor Raúl Oyola Romero" de la Universidad Nacional de Educación UGEL $N^{\circ}$ 06, llegando a las siguientes conclusión, la correlación entre inteligencia espacial y el aprendizaje en el área de arte es baja $r=0,030$. Cuando hablamos del desarrollo de capacidades lo tratamos como un resultado de un conjunto de intervenciones desde distintas áreas en el proceso cognitivo del alumno.

Lo cual concuerda con los hallazgo de nuestra investigación, en donde una variedad de materiales presentados, tanto atención visual, atención auditiva, coordinación ojo-mano, trabajos corporales, y de análisis tridimensionales favorecen la capacidad que tiene la alumna y por ende lograr un mejor desempeño en su aprendizaje en las distintas materias que se le presente. Así mismo Guevara (2012), en su tesis de investigación titulada Instrumento para el estudio de la percepción, su aplicación a la arquitectura y la lectura del espacio, donde la hipótesis a confirmar y corroborar es de que la percepción es el enlace atemporal entre el hombre y el espacio, es el medio vinculante que hace posible la experiencia cognoscitiva y entendimiento de su mundo físico existente, que por medio del control de estas se adquiere la posibilidad de poder manipular los sentidos, para mejor control de los elementos y las variables que intervienen en el diseño y la creación del espacio.

El autor aborda en la primera sección de su investigación el enfoque psicológico acerca de la percepción como parte de la demostración de la hipótesis de la tesis, ya que esta disciplina la ha considerado dentro de sus principales motivos de estudio definiéndola en términos generales como el proceso cognitivo de la conciencia del hombre capaz de reconocer e interpretar sensaciones del mundo físico, en las que intervienen otros procesos psicológicos entre los que se encuentran el aprendizaje y la simbolización, de acuerdo con nuestra investigación podemos afirmar que el programa de atención diseñado para el desarrollo perceptivo en las estudiantes, ayuda favorablemente en el desarrollo cognitivo y por ende facilita el proceso de enseñanza aprendizaje en las estudiantes.

Así mismo tenemos que reconocer que dentro de la malla curricular emitida por el Ministerio de Educación se prioriza una gran cantidad de horas para el dictado de las áreas de lógico - matemático, así como de Comunicación Integral, imposibilitando el desarrollo de otras habilidades en los estudiantes, haciéndoles entender internamente que a todos se les prepara para carreras de ingeniería así como carrera de letras, aun siendo vigente teorías donde nos refieren la existencia 
de dos tipos de inteligencia y por consiguiente la etiquetación de los estudiantes respectos a sus capacidades.

De esta manera el sistema aparentemente refiere que se adecua a los nuevos enfoques de estudios de las inteligencias pero solo de palabras y no en hechos que puedan favorecer a los estudiantes y de esta manera no adecuándose el sistema a los nuevos avances de la ciencia no poniéndolo en práctica sin dar la prioridad a las diferentes habilidades que posee cada estudiante, porque debemos entender que un aula no es homogénea, sino más bien es heterogénea y cada uno tiene habilidades diferentes.

Es necesario reconocer que existen individuos como Albert Einstein que utilizan sus inteligencias para solucionar problemas, pero que en sus centros de estudios son rechazados y duramente cuestionados por tener dificultades en sus aprendizajes, pero que posteriormente demostraran sus capacidades.

Basadas en estas reflexiones, así mismo por lo que decía el P.D. Dr Cacabelo en su última ponencia en el 2016 realizada en la Universidad Continental, sobre las bases genómicas de la demencia, es necesario trabajar una cultura preventiva, y que Sudamérica como mundo joven no debe de dejar a la niñez y se debe trabajar desde antes de diagnosticar el caso, es por eso que me planteo ¿̇Cómo influye el programa atención en el desarrollo perceptivo de los estudiantes varones y mujeres en el nivel inicial?, esto nos trae a replantear si las actividades deberán bajar algunas dificultades por la edad; y plantearnos nuevas hipótesis de investigación.

Finalmente invitar a los jóvenes investigadores a abordar el tema de percepción y atención, para contribuir con nuevas investigaciones que permiten conocimientos y aportes científicos.

En conclusión:

1. Los resultados corresponden por lo tanto que el nivel de significación sig. $=0,000$, es menor que el $\alpha=0,05$, lo que permite rechazar la hipótesis nula de igualdad de medias de la prueba del pre-test y postest, llegando a la conclusión que el programa atención genera una influencia favorable con un nivel de significación unilateral del $5 \%$ en el desarrollo perceptivo en estudiantes de la Institución educativa N 30054 Santa María Reyna Huancayo.

2. Con un nivel de significancia unilateral del $5 \%$ el programa de atención influye favorable y significativamente en el desarrollo de la rapidez perceptiva.
3. Con un nivel de significancia unilateral del $5 \%$ el programa atención influye favorable y significativamente en el desarrollo de la inteligencia espacial.

\section{Agradecimiento}

A la Institución Educativa Santa María Reyna, a nombre de su Director, a los maestros y maestras de ayer y hoy y a todas las estudiantes del 3ro. de primaria, por el apoyo brindado para la realización de la investigación.

\section{REFERENCIAS BIBLIOGRÁFICAS}

Castellanos, J. (2015). Intervención en el aula para la mejora de la atención y el rendimiento en el alumno de segundo nivel de educación primaria. Eficacia de autoinstrucción y autoobservación. (Tesis Doctoral). Universidad de Valencia.

Coon, D. (1998). Psicología. Exploraciones y aplicaciones. (8va. Edición). México: Internacional Thomson Editores.

Goldstein, B. (2005). Sensación y percepción. (6ta. Edición). México: Thomson Editores S.A. de C.V.

Guevara, A. (2012). Instrumentos para el estudio de la percepción, su aplicación a la arquitectura y la lectura del espacio. (Tesis de Maestro). Universidad de Nuevo León.

Huerta \& otros (2010), Las inteligencias múltiples y el aprendizaje de las diversas áreas curriculares en los estudiantes del 4to. Y 5to. Ciclo de primaria del colegio experimental "Víctor Raúl Oyola Romero" de la Universidad Nacional de Educación UGEL N ${ }^{\circ}$ 06. Universidad Nacional de Educación Enrique Guzmán y Valle La Cantuta.

I.E. Santa María Reyna (2016). Proyecto educativo institucional. Institución Educativa Santa María Reyna. Huancayo-Perú.

Lara, L. (2004). Utilización de un ordenador para el desarrollo de la visualización espacial. (Tesis Doctoral). Universidad Complutense de MadridEspaña.

MINEDU (2016). Evaluación ECE 2016- Informe ECE por Regiones. http://umc.minedu.gob.pe/resultadosece2016/

Sierra, R. (2007). Tesis doctorales y trabajos de investigación científica. (5ta. Edición). España: Thomson Editores Spain Paraninfo S.A.

Triola, M. (2012). Estadística. (Decimoprimera impresión). México: Editorial Pearson Educación.

Thurstone, L. \& Yela, M. (1979). Test de Percepción de diferencias. (3ra. Edición). España: TEA Ediciones S.A. 
UNESCO (2011). Compendio mundial de la Educación. Recuperado de: http://vis.unesco.org/ sites/default/files/documents/global-educationdigest-2011-comparing-education-statisticsacross-the-world-sp.pdf

Verdugo, T. García, L. \& Portillo, S. (2014). Inteligencia espacial y su relación con el rendimiento académico en niños escolarizados de 10-12 años.
Revista electrónica de investigación educativa Sonorense, Año VI, No 16 Diciembre. P.p. 9-31.

Vizcarra, C. (2012). Organización perceptiva y escritura cursiva en primer grado de primaria de tres Instituciones educativas del Callao. (Tesis de Maestría). Universidad San Ignacio De Loyola. Lima Perú. 